\title{
Determinación del desplazamiento lateral de vehículos en carreteras urbanas de Costa Rica
}

\section{Determination of vehicle wheel wander for urban roadways in Costa Rica}

\section{Velvet Morris Mitchell}

Universidad de Costa Rica, San Pedro de Montes de Oca, San José, Costa Rica.

velvetmorrism@yahoo.com

\section{Fabricio Leiva Villacorta}

National Center for Asphalt Technology, Auburn University, $A L$, USA.

leivafa@auburn.edu

Fecha de recepción: 20 de junio del 2016 / Fecha de aprobación: 20 de agosto del 2016

\section{RESUMEN}

El objetivo del proyecto consiste en determinar un valor de desplazamiento lateral que sea representativo para carreteras urbanas de Costa Rica, que pueda ser utilizado para el diseño y simulación del deterioro de pavimentos. Para esto se realizó un cálculo del desplazamiento lateral en 6 diferentes estaciones ubicadas en rutas nacionales dentro del Gran Área Metropolitana de Costa Rica con características similares.

El desplazamiento lateral es la dispersión de los vehículos en el ancho del carril, estos usualmente tienen una distribución de tipo normal. En los puntos en los que transitan más vehículos hay mayor deterioro del pavimento, por lo tanto, entre mayor es el desplazamiento lateral los vehículos se distribuyen más en el carril y el daño al pavimento es menor.

Las estaciones donde se realizaron las mediciones se ubican en la Ruta Nacional 3, Ruta Nacional 2, Ruta Nacional 202, Ruta Nacional 32, Ruta Nacional 39 y Ruta Nacional 108. Los cuales fueron seleccionados por tener un alto porcentaje de vehículos pesados. En cada estación se colocó una cámara y se trazaron líneas para medir la posición transversal en el carril de los vehículos que transitaban en el sitio, tanto livianos y pesados.

Se calculó el desplazamiento lateral utilizando la ecuación de la desviación estándar de los datos de la posición de los vehículos, y el intervalo de confianza utilizando la ecuación del intervalo de confianza de la desviación estándar. Los resultados muestran el valor obtenido es significativamente mayor que el valor de desplazamiento lateral de $10 \mathrm{~cm}$ utilizado por el LanammeUCR para los ensayos de simulación del deterioro de pavimentos.

PALABRAS CLAVES: desplazamiento lateral, pavimentos flexibles, Guía Mecanístico-Empírica para el Diseño del Pavimentos.

\section{ABSTRACT}

The main objective of this project consists in estimate a value of wheel wander representative of Costa Rican roadways, which could be used for pavements design and testing. In order to achieve the objective, wheel wander was measured and computed in 6 different stations with similar characteristics located in national routes from the Great Metropolitan Area of Costa Rica.

Wheel wander is the scatter of vehicles along the length of the lane. Drivers usually follow a normal distribution pattern. In the wheel paths that most drivers follow, traffic load will be higher causing rutting in the pavement. Therefore, in roadways where wheel wander is high, the traffic load is distributed along the lane width, increasing the design life.

For each station only the outer lane, the closest to the camera, was used. These stations are located in National Route 3, National Route 2, National Route 202, National Route 32, National Route 39 and National Route 108.

These routes were chosen because of their high truck traffic volume. In each station, a camera was placed roadside. Also lines were drawn covering more than the half of the lane cross section. The position of both heavy and light vehicles was measured.

Standard deviation and confidence interval for variance equations were used to determine the wheel wander. Results show that the mean and 95\% confidence interval of wheel wander is drastically higher that the value of $10 \mathrm{~cm}$ used by the LanammeUCR for accelerated pavements testing.

KEY WORDS: wheel wander, flexible pavements, Mechanistic-Empirical Pavement Design Guide. 


\section{1- INTRODUCCIÓN}

La importancia del desplazamiento lateral radica en que mitiga la densificación y el hundimiento, porque reduce la cantidad de vehículos sobre la huella y se aumentan a los lados de esta (Oscarsson, 2011). Se reduce la carga sobre un mismo punto, por lo que aumenta la vida útil del pavimento. Sin embargo, el valor utilizado actualmente en Costa Rica es de estudios realizados en otros países, por lo que el desgaste real de la carretera puede ser diferente de cómo se modela.

Entonces al determinar este parámetro, se puede realizar diseños y simulaciones que se aproximen mejor a la realidad. Lo cual puede producir beneficios económicos en la construcción de pavimentos, principalmente asfalticos.

Los valores medidos en otras carreteras pueden no ser representativos para este país, debido a que depende de varios factores como las condiciones de tráfico, el clima y los conductores (Stemphihar, Williams, \& Drummer, 2005).

Adicionalmente, conocer el desplazamiento lateral tiene diferentes beneficios en el diseño de pavimentos y en ensayos a escala natural. Este puede ser implementado para el simulador de vehículos pesados (HVS por sus siglas en inglés) del LanammeUCR de la Universidad de Costa Rica. El HVS se utiliza para evaluar el rendimiento de los pavimentos simulando en pocos meses las condiciones de tránsito a las que estará sometido en varios años. El HVS requiere del desplazamiento lateral como parámetro de entrada, actualmente se utiliza un valor de $10 \mathrm{~cm}$ (Leiva, Aguiar, \& Loría, 2015). Este valor se basa en estudios previos realizados por investigadores y operadores de equipos similares al HVS. Por lo que al determinar el promedio de desplazamiento lateral para Costa Rica se puede tener una mejor aproximación del comportamiento real de los vehículos en las carreteras del país. Además, de utilizar este valor para la simulación, puede implementarse para el diseño de pavimentos en una futura guía de diseño de pavimentos en Costa Rica.

El objetivo de este estudio consiste en determinar un valor de distribución de la desviación lateral representativo para las carreteras en Costa Rica con el fin de generar valores de desplazamiento lateral para usar en el diseño de pavimentos en Costa Rica.

\section{2- METODOLOGÍA}

El procedimiento realizado consiste en un análisis del efecto del desplazamiento lateral sobre el pavimento, la medición de la posición de los vehículos en el carril, un análisis probabilístico de los datos y el cálculo del desplazamiento lateral.

\subsection{Análisis de sensibilidad MEPDG}

Para examinar el efecto del desplazamiento lateral en este informe se utilizó la Guía para el Diseño Mecanístico-Empírica de estructuras de Pavimentos para carreteras nuevas y rehabilitadas, la cual fue realizada por el Programa Cooperativo Nacional de Investigación de Autopistas de los Estados Unidos (NCHRP por sus siglas en inglés), la Junta de Investigación del Transporte (TRB por sus siglas en inglés) y el Consejo Nacional de Investigación de Estados Unidos (NRC por sus siglas en inglés).

Al mantener todos los demás valores constantes y modificar el desplazamiento lateral, se puede observar el efecto producido únicamente por este último sobre el pavimento en el agrietamiento tipo piel de cocodrilo, la deformación permanente y el Índice de Rugosidad Internacional (IRI) a lo largo del tiempo.

Los parámetros utilizados son los establecidos en el ejemplo de Concreto Asfáltico Convencional (AC por sus siglas en inglés) que contiene la guía. Este ejemplo se seleccionó debido a que todas las carreteras analizadas en este proyecto son de asfalto. Además, es el tipo de pavimento más utilizado en el país. Y los valores de desplazamiento lateral que se introdujeron al programa son 10 $\mathrm{cm}, 15 \mathrm{~cm}, 20 \mathrm{~cm}, 25 \mathrm{~cm}, 30 \mathrm{~cm}, 35 \mathrm{~cm}$ y $40 \mathrm{~cm}$.

\subsection{Método de medición}

El método consiste en utilizar una plantilla para dibujar líneas a una distancia específica con pintura blanca en la carretera. Las líneas son de $20 \mathrm{~cm}$ de longitud, de $2,5 \mathrm{~cm}$ de ancho y se encuentran separadas a $10 \mathrm{~cm}$ de la siguiente. Con estas medidas la incertidumbre es de $6,25 \mathrm{~cm}$, el cual es la mitad de la distancia entre el centro de dos líneas consecutivas.

El punto de referencia de la medición empieza donde termina la línea del margen de la carretera que delimita el carril, de forma que respecto a esta línea y la primera dibujada hay $10 \mathrm{~cm}$. En todos los casos se utilizaron los carriles externos porque se requiere que otros vehículos no obstruyan la grabación de la cámara. Del mismo modo, se eligieron sitios con pocos carriles, para evitar que los conductores cambiaran de carril al ver las marcas.

Para determinar los datos se consideró como si el borde externo de la rueda estuviese ubicado en el centro de la línea más cercana. A este valor se le sumó un valor $\Delta$, que corresponde a la mitad del ancho del neumático para los vehículos livianos y la distancia entre el borde del neumático al centro de las dos llantas para los pesados, como se observa en la Figura 1. 

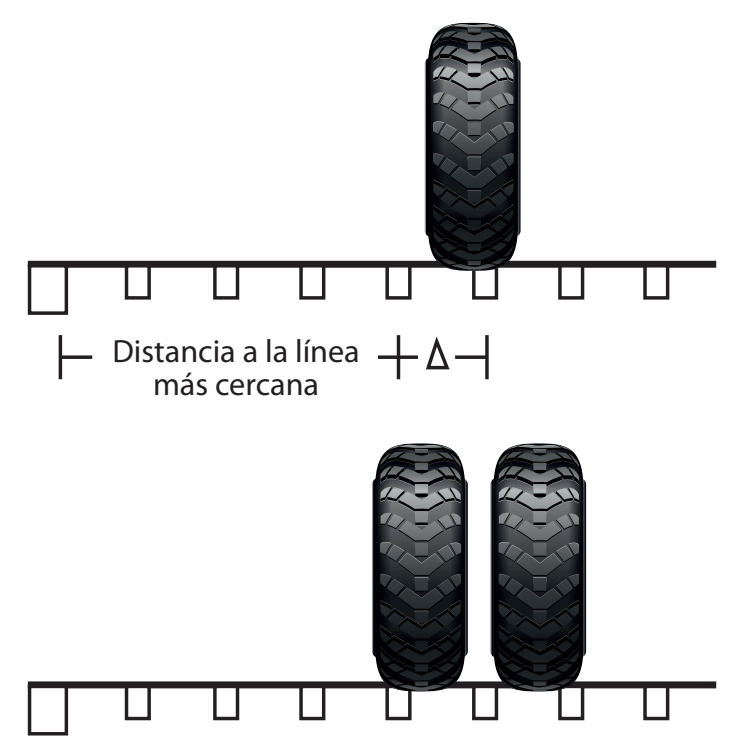

$\vdash \underset{\text { más cercana }}{\text { Distancia la lín }}+\Delta \dashv$

Figura 1. Método para medir la distancia del neumático respecto al margen para vehículos livianos y pesados.

La distancia adicional al centro del neumático se consideró como 6,25 cm. Porque 12,5 cm es el ancho de banda (ancho de la parte en contacto con el pavimento) promedio en el catálogo de llantas para vehículos livianos que se utilizó como referencia (REENFRIO, 2015).

En cuanto a vehículos pesados, como se consideraron únicamente vehículos con más de dos llantas en el eje trasero, la distancia se debe medir al centro de las dos llantas, la cual es mayor al ancho de banda del neumático. Se intentó buscar un ancho promedio para cada tipo de eje. Sin embargo, estas distancias no varían significativamente entre los diferentes modelos por lo que se utilizó el mismo valor para vehículos de eje simple llanta doble, tándem y trídem, el cual es de 33,9 cm (Diesel San Miguel, 2015).

\subsection{Ubicación}

Se utilizaron seis estaciones de muestreo ubicadas en carreteras dentro del Gran Área Metropolitana (Figura 2), en las cuales transitan una cantidad de vehículos pesados significativos (TPD de camiones mayor que 1000 vehículos por día). Las carreteras seleccionadas son las Rutas Nacionales 3, 2, 202, 32, 39 y 108.

Para disminuir errores en los resultados debido a los factores que afectan el desplazamiento lateral se escogieron zonas con características similares. La mayoría de los sitios tienen un ancho del carril entre 3,45 y 3,65 m, y máximo dos carriles en el mismo sentido para evitar errores de medición debido a vehículos cambiando carriles.
Tampoco se incluyeron los vehículos que por algún motivo cambiaron su trayectoria. Esto incluye a los conductores que intentaron esquivar las marcas en la carretera o automóviles afectados por buses estacionados.

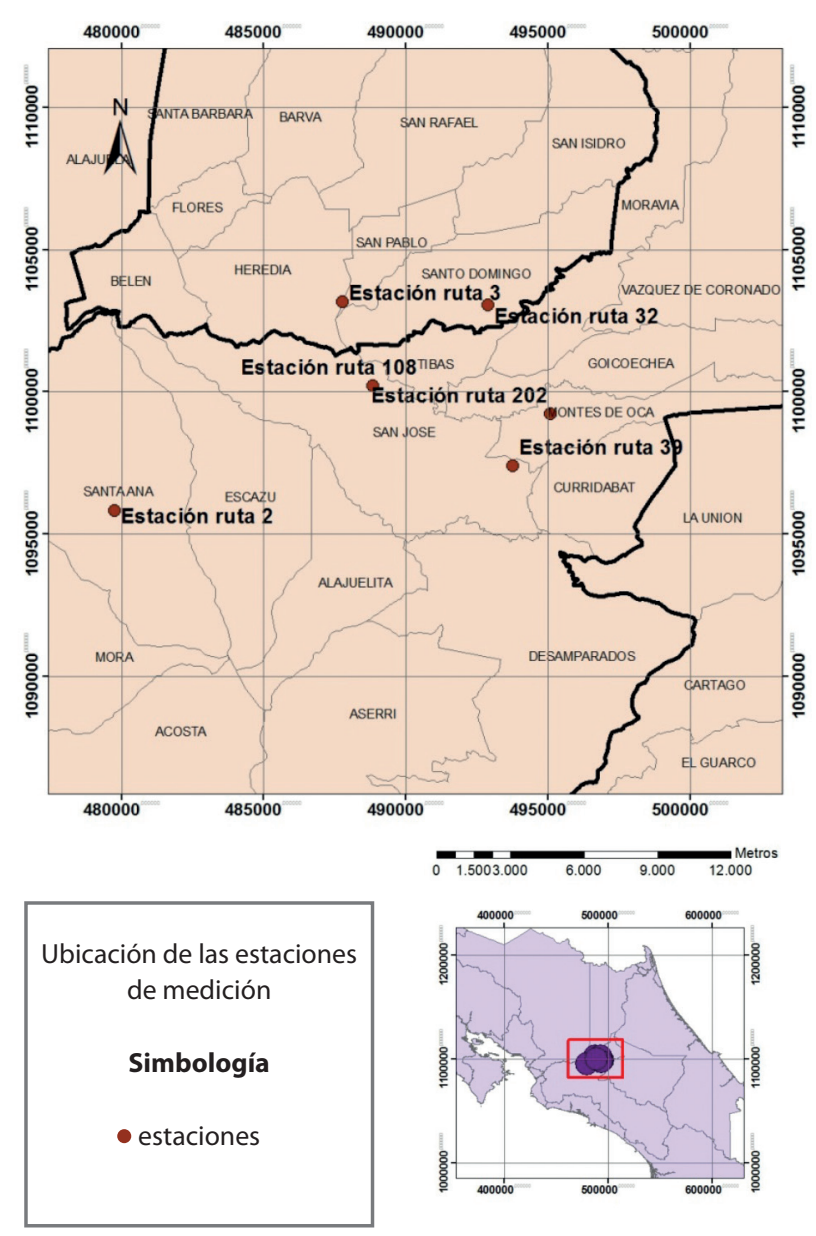

Figura 2. Mapa de ubicación de las estaciones de medición.

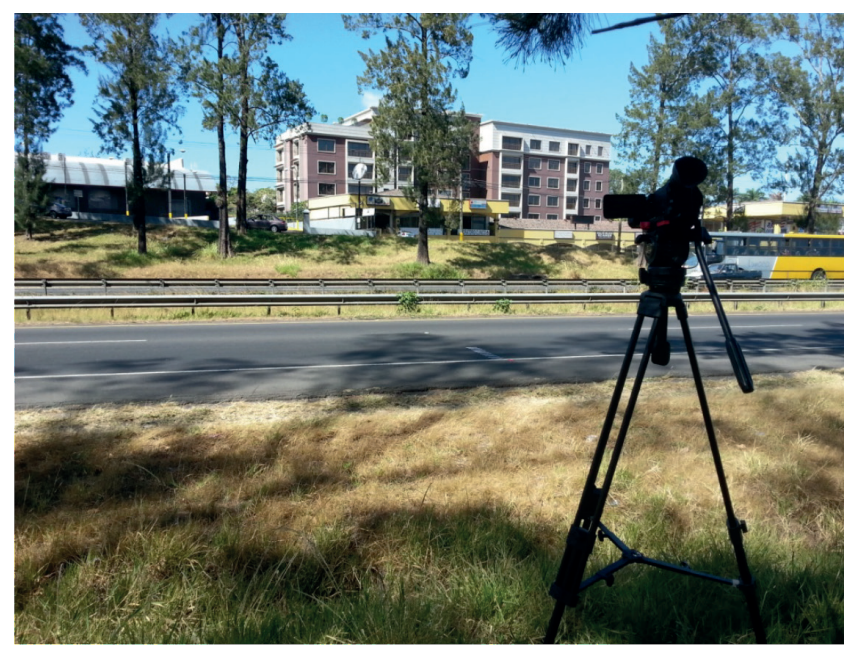

Figura 3. Fotografía de la ubicación de la cámara en la estación de la Ruta Nacional 2. Tomada el 7 de abril del 2015. 


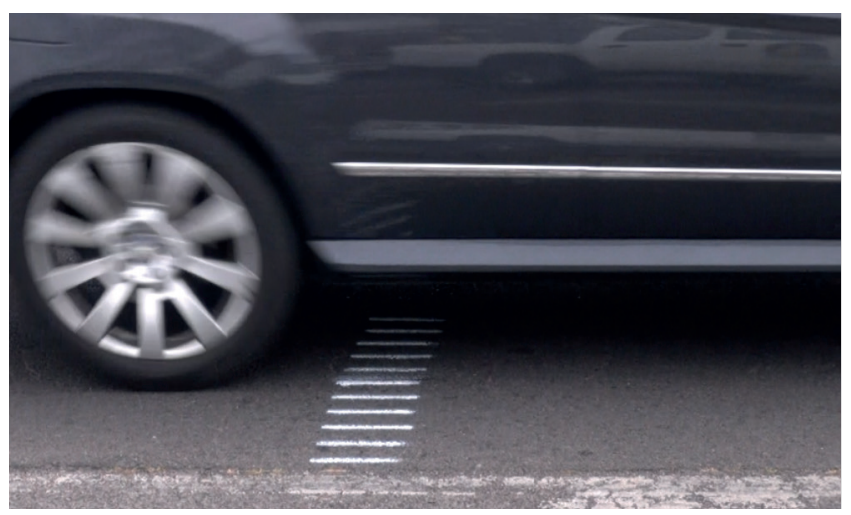

Figura 4. Fotografía de un vehículo transitando sobre las líneas de medición en la Ruta Nacional 39.

Tomada el 12 de mayo del 2015.

\subsection{Modelo estadístico}

En este estudio, se utilizó una prueba de hipótesis para demostrar si los datos obtenidos en campo siguen una tendencia normal $u$ otra distribución. La hipótesis nula se escoge en la prueba para cada una de las distribuciones suponiendo que los datos siguen la distribución. Esto es lo que se busca comprobar. Estas hipótesis se definen a continuación:

- Hipótesis nula (H0): Los datos tienen tendencia de la distribución especificada.

- Hipótesis alternativa (Ha): Los datos no tienen tendencia de la distribución especificada

La prueba de Anderson-Darling es lo que permitirá rechazar o fallar en rechazar la hipótesis nula. Para esto se utilizó determinó el valor P con el programa Minitab. El valor P o nivel observado de la significancia es un método para determinar una conclusión de la prueba de hipótesis. Si se supone la hipótesis nula como verdadera, el valor $\mathrm{P}$ es la probabilidad de que una muestra se encuentre en el rango de datos para que se cumpla lo establecido por H0. Si el valor $\mathrm{P}$ es menor que la significancia, que en este caso es 0,05 , se rechaza la hipótesis nula (Anderson, Sweeney, \& Williams, 2004).

\section{3- RESULTADOS}

\subsection{Resultados del análisis de sensibilidad con el programa MEPDG}

El efecto de diferentes valores de desplazamiento lateral en el agrietamiento, según el programa MEPDG, se muestran en Figura 5. Para el menor valor evaluado, $10 \mathrm{~cm}$ (o 4 in como se introdujo en el programa), el porcentaje de agrietamiento en 20 años es cercano a $50 \%$ del área. Mientras que para el valor mayor
(30 cm o 12 pulgadas), es de un 35,56 \%. La diferencia respecto al mayor agrietamiento es $11,87 \%$.

El límite de agrietamiento recomendado por la guía es de $25 \%$. Este valor es sobrepasado entre los años ocho y nueve cuando el desplazamiento lateral es de $10 \mathrm{~cm}$. Y entre los años 12 y 13, cuando es de $30 \mathrm{~cm}$. Esto muestra que la vida útil del pavimento puede aumentar o reducirse varios años según el desplazamiento lateral.

Al igual que el agrietamiento, la deformación permanente o ahuellamiento hay un mayor daño entre mayor es el desplazamiento lateral como se muestra en la Figura 6.

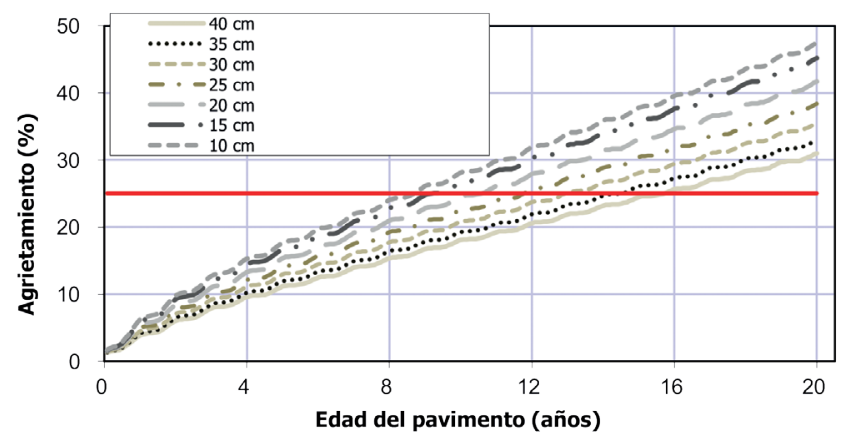

Figura 5. Variación temporal en el porcentaje de agrietamiento tipo piel de cocodrilo para diferentes desviaciones laterales. Los datos fueron estimados con la Guía mecanísticoempírica de diseño de pavimentos

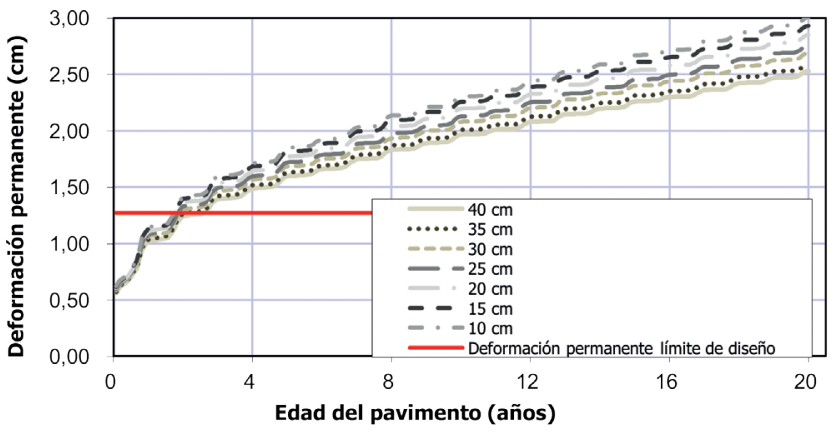

Figura 6. Variación temporal de la deformación para diferentes desviaciones laterales estimada con la Guía mecanístico-empírica de diseño de pavimentos.

El IRI o Índice de Rugosidad Internacional es la desviación vertical del perfil de un pavimento de la forma en que fue diseñado (Secretaría de Integración Económica Centroamericana (SIECA), 2002). Se mide en metros entre kilómetro o en pulgadas entre milla. Este índice se utiliza para medir la regularidad en una carretera, y afecta la velocidad de tránsito, consumo de combustible y deterioro de vehículos además de las deformaciones del pavimento (Badilla, 2009). Puesto que el IRI se ve afectado por la deformación permanente también por el desplazamiento lateral. El efecto del cambio de este último en el IRI se puede observar en la Figura 7. Para el IRI, al igual que la deformación permanente y 
el agrietamiento, el IRI aumenta una mayor cantidad en menor tiempo conforme el desplazamiento lateral es más bajo. Sin embargo, la diferencia es menos perceptible.

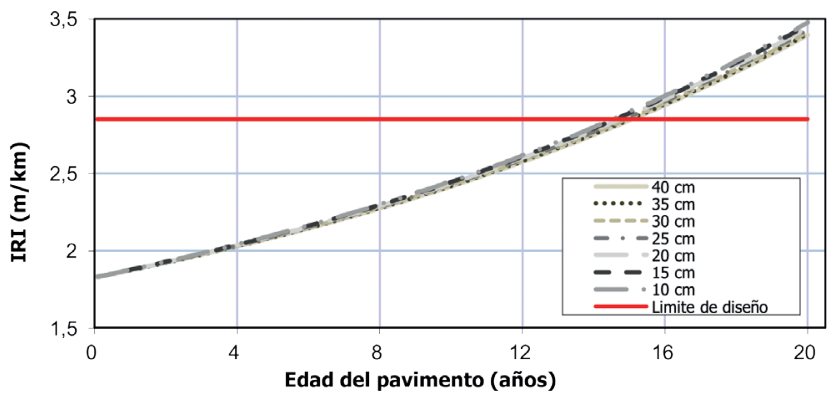

Figura 7. Variación temporal en el Índice de Rugosidad Internacional (IRI) para diferentes desviaciones laterales estimado con la Guía mecanísticoempírica de diseño de pavimentos

\subsection{Resultados de desplazamiento lateral}

La Figura 8 presenta la frecuencia acumulada de los vehículos pesados para todas las rutas nacionales. El comportamiento es similar en la ruta nacionales 2 y 3, donde el $50 \%$ se ubica a 100 cm como máximo del carril. En la curva de la Ruta Nacional 32, aproximadamente un $70 \%$ transita a menos de $100 \mathrm{~cm}$ del borde. Mientras que en la Ruta Nacional 39 sólo el 40 \% transita a esta distancia. Las rutas nacionales 108 y 202, presentan tamaños de carril significativamente menor y mayor, respectivamente, por lo que las curvas son diferentes.

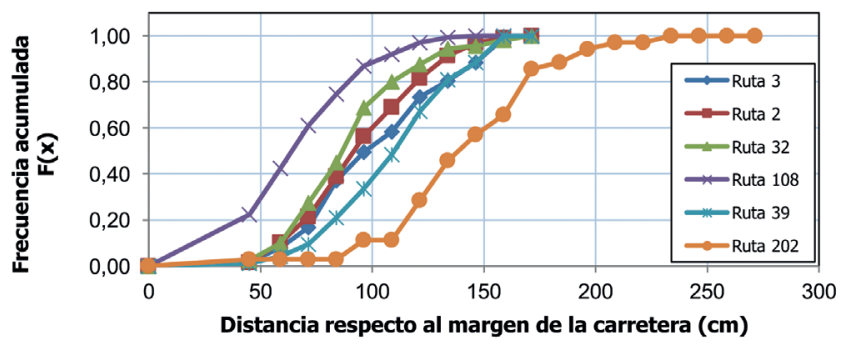

Figura 8. Frecuencia acumulada de la ubicación del neumático doble respecto al margen de la carretera para vehículos pesados.

En la mayoría de las rutas nacionales estudiadas, excepto en la ruta 202 para eje simple llanta doble y vehículos pesados, el valor $\mathrm{P}$ es menor que la significancia. Esto significa que los datos no tienen tendencia normal.

El promedio de la desviación estándar o desplazamiento lateral es de 28,11 cm para vehículos livianos y 29,05 cm para los pesados en las estaciones muestreadas, como se muestra en el Cuadro 1. El promedio para ambos tipos es similar, lo cual coincide por lo establecido por Stemphihar, Williams y Drummer (2005) que el tipo de vehículo no influye significativamente. Esto se refuerza con la desviación estándar promedio obtenida para los vehículos de eje simple llanta doble y tándem que tienen valores similares, 29,59 y 31,56 respectivamente.

Cuadro 1. Valores de desplazamiento lateral por tipo de vehículo y de eje para todas las rutas nacionales muestreadas.

\begin{tabular}{|c|c|c|c|c|c|}
\hline \multirow{2}{*}{ Estaciones } & \multicolumn{5}{|c|}{ Desplaramiento lateral (cm) } \\
\cline { 2 - 6 } & Livianos & Pesados & & & \\
\cline { 2 - 6 } & Doble & Tándem & Trídem & Todos \\
\hline $\begin{array}{c}\text { Ruta Nacional 3 } \\
\text { (Heredia) }\end{array}$ & 25,30 & 31,11 & 31,92 & 42,14 & 31,36 \\
\hline $\begin{array}{c}\text { Ruta Nacional 2 } \\
\text { (Cartago) }\end{array}$ & 26,82 & 27,71 & 28,90 & 7,22 & 27,56 \\
\hline $\begin{array}{c}\text { Ruta Nacional 202 } \\
\text { (Sabanilla) }\end{array}$ & 42,87 & 33,75 & 50,93 & 0 & 36,48 \\
\hline $\begin{array}{c}\text { Ruta Nacional 32 } \\
\text { (Guápiles) }\end{array}$ & 25,01 & 31,13 & 25,17 & 17,37 & 26,58 \\
\hline $\begin{array}{c}\text { Ruta Nacional 39 } \\
\text { (Zapote) }\end{array}$ & 20,16 & 28,26 & 30,02 & 12,50 & 27,79 \\
\hline $\begin{array}{c}\text { Ruta Nacional 108 } \\
\text { (La Uruca) }\end{array}$ & 28,47 & 25,59 & 22,43 & 19,24 & 24,53 \\
\hline Promedio & 28,11 & 29,59 & 31,56 & 19,69 & 29,05 \\
\hline
\end{tabular}

Sin embargo, los camiones de eje trídem tienen un valor de 19,69 cm. Esto se podría despreciar si se considera que la cantidad de muestras de este tipo de vehículos en la mayoría de las estaciones son muy pequeñas en comparación con los otros tipos, lo cual coincide con los datos del MOPT del conteos en carreteras donde los camiones de cuatro ejes son escasos (Dirección de Planificación Sectorial del MOPT, 2015). Sin embargo, en la Ruta Nacional 32, en donde la cantidad de vehículos de ejes trídem es de 39 , el desplazamiento lateral es similar al promedio para este tipo de eje.

Todos los valores de desplazamiento lateral son menores al utilizado por el Laboratorio de Pavimentos (PaveLab) del LanammeUCR que es de $10 \mathrm{~cm}$ (Leiva, Aguiar, \& Loría, 2015). Como se puede observar en la Figura 5 y la Figura 6, el agrietamiento y la deformación permanente de un pavimento con un desplazamiento lateral de $10 \mathrm{~cm}$ alcanza de dos a cuatro años antes (para los parámetros utilizados en este informe) el estado límite recomendado por la Guía de Diseño Mecanístico Empírico y por el Catálogo de Daños. En cuanto al IRI (Figura 7) y el índice de serviciabilidad, la diferencia es menor.

La Figura 9 y la Figura 10 muestran el intervalo de confianza para cada una de las rutas nacionales. El cuál es el rango en el probablemente de ubique la media de la población en las rutas nacionales para un nivel de confianza de 95\%. Hay que considerar que únicamente la Ruta Nacional 202 tiene comportamiento normal, por lo que el porcentaje de error debe ser mayor para las otras rutas. 


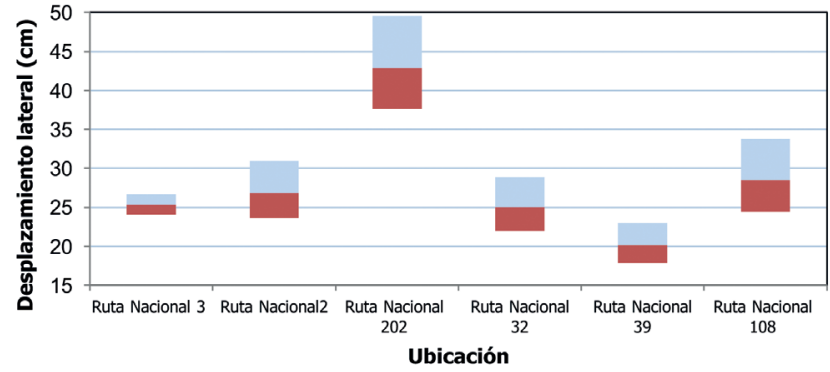

Figura 9. Desplazamiento lateral e intervalo de confianza a 95\% en vehículos livianos para todas las rutas nacionales analizadas.

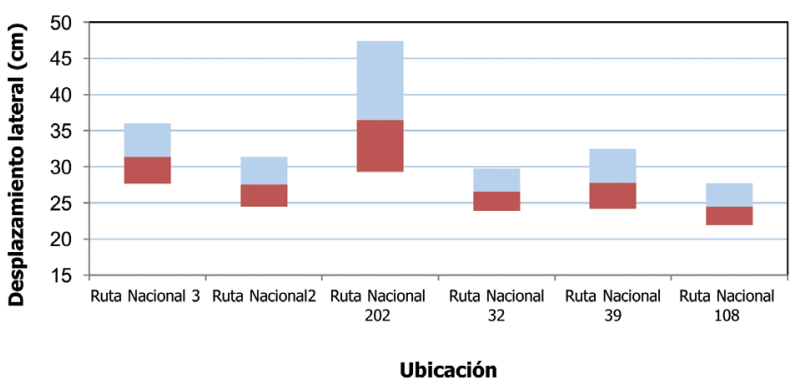

Figura 10. Desplazamiento lateral e intervalo de confianza a 95\% en vehículos pesados para todas las rutas nacionales analizadas.

Si se analizan individualmente para cada ruta nacional, en se observa que los vehículos pesados tienen un desplazamiento lateral mayor en las rutas nacionales 3 y 39. En las rutas nacionales 2 y 32 la diferencia es poco significativa. $Y$ en las rutas 202 y 108, los vehículos livianos tienen un desplazamiento lateral mayor. Estas dos tienen los carriles más anchos y angostos, respectivamente, entre las seis estaciones.

En los vehículos livianos la mayoría de los intervalos se encuentran entre 20 y $35 \mathrm{~cm}$. Excepto la parte inferior del intervalo de la Ruta Nacional 39 y la ruta 202. En la Ruta 202 el ancho del carril es mayor por lo que hay mayor espacio para transitar. Mientras que en la Ruta Nacional 39 los vehículos livianos tienden a distribuirse menos en el carril, a pesar de que el ancho del carril de esta carretera es similar a la mayoría de las rutas.

En cuanto a los vehículos pesados, la ruta 202 también sobresale por los mismos motivos mencionados anteriormente. Los intervalos de la mayoría de las rutas se ubican entre 24 y $35 \mathrm{~cm}$, el cual es similar al rango donde se ubican la mayor parte de los vehículos livianos.

El intervalo de confianza de camiones para la Ruta Nacional 108 se encuentra por debajo de los otros rangos. En esta carretera el ancho de carril es de tres $\mathrm{m}$, por lo que hay menos espacio para que se distribuyan los vehículos. Sin embargo, esto no afectó los vehículos livianos en esta ruta.
El valor de desplazamiento lateral utilizado por el LanammeUCR para el simulador de vehículos pesados de $10 \mathrm{~cm}$ (Leiva, Aguiar, \& Loría, 2015) no se encuentra dentro de ninguno de los rangos obtenidos y es inferior a todos los valores obtenidos.

\section{4- CONCLUSIONES}

Las conclusiones que se determinaron en este trabajo se muestran a continuación:

- El desplazamiento lateral de vehículos pesados promedio para carreteras del Gran Área Metropolitana de Costa Rica es 29,05 cm. El desplazamiento lateral en 4 de las 6 rutas nacionales se ubica en un rango entre 24 y $35 \mathrm{~cm}$. La incertidumbre de la medición es $\pm 6,25 \mathrm{~cm}$.

- Entre menor sea el desplazamiento lateral en una carretera mayor es el deterioro producido en el pavimento por agrietamiento tipo piel de cocodrilo, deformación permanente y rugosidad.

- Los valores determinados de desplazamiento lateral y los intervalos de confianza en este informe son mayores al utilizado por el LanammeUCR de $10 \mathrm{~cm}$ en los ensayos de simulador de vehículos pesados. Lo cual significa que los daños por agrietamiento y la deformación permanente son mayores en los ensayos del simulador de vehículos pesados que en las carreteras nacionales.

- El desplazamiento lateral no se ve afectado significativamente por el tipo de vehículo ni el tipo de eje, el rango de valores promedio determinados en este informe se encuentra entre 28,11 a 31,56 cm. En este rango no se consideró el eje trídem por la falta de muestras.

- La ubicación promedio del centro del neumático respecto al margen, tanto para vehículos livianos como para pesados, es de aproximadamente $100 \mathrm{~cm}(1 \mathrm{~m})$ para los carriles de ancho cercano a 3,6 m. La cual es la posición del neumático cuando el vehículo transita por el centro del carril.

- En la mayoría de los casos, los datos de ubicación en el carril no se ajustan a ninguna de las distribuciones probadas. Sin embargo, en algunas ocasiones como la Ruta Nacional 202 los datos siguen la distribución normal.

\section{5- RECOMENDACIONES}

- Para realizar mediciones del desplazamiento lateral utilizado el método de muestreo de líneas de referencia, se recomienda marca las líneas a lo largo de todo el carril. Esto con el fin de 
disminuir errores en el resultado producto de conductores que intenten esquivar las líneas en la carretera. También utilizar carreteras con la menor cantidad posible de carriles en el mismo sentido debido a que varios conductores cambiaron de carril al ver las líneas pintadas en la carretera.

- El método se puede modificar para colocar las líneas digitalmente, siempre que se considere la distorsión producida por la inclinación de la cámara. Con el fin de mejorar la precisión de los resultados.

- Se puede pintar líneas con una menor distancia entre sí para disminuir la incertidumbre. Sin embargo, se requieren varias muestras más para poder determinar la tendencia.

- Para analizar individualmente el comportamiento de camiones con eje trídem se recomienda realizar varias mediciones en diferentes puntos de la Ruta Nacional 32.

- Se pueden implementar los valores obtenidos en el simulador de vehículos pesados (HVS por sus siglas en inglés) e incorporar en una futura guía de diseño de pavimentos para la Gran Área Metropolitana.

\section{6- REFERENCIAS BIBLIOGRÁFICAS}

1. Agresti, A., \& Finlay, B. (1999). Statistical methods for the social sciences. New Jersey: Prentice Hall.

2. Anderson, D., Sweeney, D., \& Williams, T. (2004). Estadística para administración y economía. Mexico: Thomson.

3. Ávila, T., Badilla, G., \& Aguiar, J. P. (2013). Calibración de modelo de serviciabilidad de pavimentos flexibles de AASHTO para Costa Rica. San José, Costa Rica: Programa de Infraestructura del Transporte (PITRA).

4. Badilla, G. (2009). Determinación de la regularidad superficial de pavimentos mediante el cálculo de índice de regularidad internacional (IRI): Aspectos y consideraciones importantes. San José, Costa Rica: Laboratorio Nacional de Materiales y Modelos Estructurales.

5. Blab, R., \& Litzka, J. (1995). Measurement of lateral distribution of heavy vehicles and its effect on design of road pavements. Vienna, Austria: University of Technology.

6. Consejo de Directores de Carreteras de Iberia e Iberoamérica. (2002). Catálogo de deterioros de pavimentos flexibles. México.

7. Diesel San Miguel. (2015). Camiones: Dimensiones (mm). Recuperado el Mayo de 2015, de http://dieselsanmiguel.com.ar/

8. Dirección de Planificación Sectorial del MOPT. (2015). T.P.D. Histórico de una estación de conteo vehicular. San José: Ministerio de Obras Públicas y Transporte.

9. Epidat. (2014). Distribución de probabilidad. Recuperado el Setiembre de 2014, de Dirección Xeral de Saúde Pública de la Xunta de Galicia: http://www. sergas.es

10. Fitzpatrick, C., Harrington, C., \& Knodler Jr., M. A. (2013). The effects of clear zone size amd roadside vegetation on driver behavior. Massachusetts, USA: Transportation Research Board: 93rd Annual Meeting.

11. Ledesma, A. (2015). Estimación de varianza y proporciones poblacionales mediante intervalos de confianza. Obtenido de Facultad de Ingeniería Universidad de la Plata: http://www.ing.unlp.edu.ar

12. Leiva, F., Aguiar, J., \& Loría, L. G. (2015). Status of the first experiment at the Pavelab. Recuperado el Noviembre de 2015, de http://www.pavetrack.com

13. Ministerio de Obras Públicas y Transporte (MOPT). (2003). Reglamento de circulación por carretera con base en el peso y las dimensiones de los vehículos de carga. San José, Costa Rica: Decreto Ejecutivo No. 31363-MOPT.

14. Minitab. (2015). Análisis de capacidad. Recuperado el Noviembre de 2015, de Soporte de minitab: http://support.minitab.com/

15. National Cooperative Highway Research Program (NCHRP). (2004). Guide for Mechanistical Empirical Design of new and rehabilitated pavement structures. IIlinois.

16. Oscarsson, E. (2011). Mechanistical-Empirical Modeling of Permanent Deformation in Asphalt Concrete Layers. Lund, Suecia: Universidad de Lund.

17. REENFRIO. (2015). Productos. Recuperado el Noviembre de 2015, de http://www.reenfriocr.com/

18. Secretaría de Integración Económica Centroaméricana (SIECA). (2000). Catálogo Centroamericano de daños a pavimentos viales. Guatemala.

19. Secretaría de Integración Económica Centroamericana (SIECA). (2002). Manual centroamericano para diseño de pavimentos. Guatemala.

20. Stemphihar, J., Williams, R. C., \& Drummer, T. (2005). Quantifying the lateral displacement of trucks for use in pavements design. Michigan: Michigan Technological University.

21. Timm, D., \& Priest, A. (2005). Wheel wander at the NCAT test track. Auburn, USA: National Center for Asphalt Technology.

22. Zuñiga, J. C., \& Trejos, J. L. (2011). Conteos vehiculares. Recuperado el Setiembre de 2014, de Ministerio de Obras Públicas y Transporte: http://mopt.go.cr/ 